\title{
Intrahepatic Bile Duct Adenoma
}

National Cancer Institute

\section{Source}

National Cancer Institute. Intrahepatic Bile Duct Adenoma. NCI Thesaurus. Code C7126.

A rare adenoma that arises from the intrahepatic biliary tree. 